\title{
Pyelonephritis in Pregnancy: Relationship of Fever and Maternal Morbidity
}

\author{
Tracey H. DeYoung, MD ${ }^{1} \quad$ Julie R. Whittington, MD ${ }^{2}$ Christopher S. Ennen, MD ${ }^{1} \quad$ Aaron T. Poole, MD \\ ${ }^{1}$ Department of Obstetrics and Gynecology, Naval Medical Center \\ Portsmouth, Portsmouth, Virginia \\ 2 Department of Obstetrics and Gynecology, University of Arkansas \\ for Medical Sciences, Little Rock, Arkansas \\ Address for correspondence Tracey H. DeYoung, MD, Department of \\ Obstetrics and Gynecology, Naval Medical Center Portsmouth, 620 \\ John Paul Jones Circle, Portsmouth, VA \\ (e-mail: Tracey.h.deyoung.mil@mail.mil).
}

Am J Perinatol Rep 2019;9:e366-e371.

\begin{abstract}
\section{Keywords}

- pyelonephritis

- pregnancy

- fever

- morbidity

- acute respiratory distress syndrome

- ARDS

- MEWC

- maternal early warning criteria

Objective To evaluate the relationship between maternal fever at the time of hospital admission and subsequent maternal morbidity in pregnant patients with pyelonephritis.

Study Design In this retrospective cohort study, inpatient records were reviewed for all obstetric patients discharged from a single tertiary care hospital between June 1 , 2011, and May 30, 2017, with the diagnosis of pyelonephritis. Patients were stratified into two groups, those with and without fever at the time of admission. Descriptive statistics were utilized to evaluate the association of fever at the time of presentation with subsequent morbidity. Using admission vital signs, maternal early warning criteria (MEWC) were applied and odds ratios calculated to predict intensive care unit (ICU) admission.

Results A total of 110 patients were admitted with pyelonephritis in pregnancy; 24 patients were febrile and 86 patients were afebrile on admission. There was no difference in rates of maternal ICU admission between both groups. Positive MEWC was predictive of ICU admission with an adjusted odds ratio of 16.54 (95\% confidence interval: $1.29-212.5 ; p=0.03$ ).

Conclusion Afebrile pregnant patients with pyelonephritis remain at risk of significant maternal morbidity. Application of the MEWC on admission identifies patients at higher risk of ICU admission.
\end{abstract}

Pyelonephritis complicates $0.5 \%$ of pregnancies. ${ }^{1}$ It is one of the most common causes of maternal sepsis, the third leading cause of maternal mortality worldwide. ${ }^{2}$ Despite interventions to decrease pyelonephritis in pregnancy, such as screening for asymptomatic bacteriuria, the incidence of pyelonephritis has remained steady. ${ }^{3}$ Acute pyelonephritis is associated with significant maternal and fetal morbidities with complications including preterm labor, preterm birth, acute respiratory distress syndrome (ARDS), sepsis, acute renal failure, and maternal or fetal death. ${ }^{1,4}$ Pyelonephritis is one of the most common nonobstetric indications for antepartum hospitalization.

Anatomic and physiologic changes to the upper and lower urinary tracts in pregnancy predispose the pregnant woman to

received

February 27, 2019

accepted after revision

July 9, 2019
DOI https://doi.org/

$10.1055 / \mathrm{s}-0039-1695746$ ISSN 2157-6998. the development of pyelonephritis. High levels of progesterone lead to smooth muscle relaxation and subsequent decreased peristalsis in the renal collecting system and ureters as well as increased bladder capacity. This, in combination with the physical obstruction secondary to the gravid uterus, leads to hydroureteronephrosis and urinary stasis. ${ }^{5}$ These factors lead to the increased rates of pyelonephritis in pregnancy, especially if asymptomatic bacteriuria is present. Additional risk factors include prior urinary tract infections, sickle cell trait, anemia, and diabetes mellitus. ${ }^{4-6}$

The diagnosis of pyelonephritis is established clinically based on symptoms of fever, flank pain, and costovertebral angle tenderness, accompanied by pyuria or bacteriuria., 3
Copyright $\odot 2019$ by Thieme Medical

Publishers, Inc., 333 Seventh Avenue, New York, NY 10001, USA. Tel: +1(212) 584-4662.
License terms

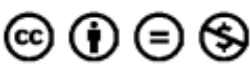


Given the potential for severe morbidity, if the diagnosis is suspected, hospitalization is recommended for treatment with intravenous antibiotics until symptomatically improved and afebrile for more than 24 hours, followed by an additional 10 to 14 days of oral antibiotics. ${ }^{5}$

Two of the most severe complications of pyelonephritis in pregnancy are sepsis and pulmonary insufficiency or ARDS, which occurs in 1.9 to $17 \%$ and 0.5 to $7 \%$ of cases, respectively. ${ }^{1,3}$ Early recognition of these complications is critical to ensure a favorable outcome; therefore, it would be useful to readily identify which patients with pyelonephritis were at higher risk of these potentially devastating complications. ${ }^{7}$ Fever is the most common presenting sign or symptom of sepsis in pregnancy; however, additional vital sign abnormalities may be present indicating more advanced case of sepsis. ${ }^{8}$ In 2014, the maternal early warning criteria (MEWC) was proposed by the Subcommittee on Vital Sign Triggers from the National Partnership for Maternal Safety. This is a single parameter risk assessment system utilized to identify patients at risk of developing acute illness who may need increased intensity or frequency of monitoring or transfer to a higher level of care. The critical triggers for the MEWC are listed in - Table $1 .^{9}$

The objective of our study was to evaluate whether fever, specifically at the time of initial evaluation, is associated with increased morbidity as indicated by ICU admission. We hypothesized that those patients with a fever at the time of initial presentation may have increased maternal morbidity. We also sought to assess the risk of sepsis, ARDS, preterm labor, and preterm delivery based on fever status at admission. Additionally, we assessed the utility of the MEWC at the time of admission to predict a later ICU admission.

\section{Materials and Methods}

We conducted an Institutional Review Board approved, singlecenter historical cohort study. All patients discharged from the obstetrics and gynecology service at an academic military hospital with the diagnosis of pyelonephritis between June 1, 2011, and May 30,2017, were identified through a query of the

Table 1 Maternal early warning criteria

\begin{tabular}{|l|l|}
\hline Systolic blood pressure $(\mathrm{mm} \mathrm{Hg})$ & $<90$ or $>160$ \\
\hline Diastolic blood pressure $(\mathrm{mm} \mathrm{Hg})$ & $>100$ \\
\hline Heart rate (beats per min) & $<50$ or $>120$ \\
\hline Respiratory rate (breaths per min) & $<10$ or $>30$ \\
\hline $\begin{array}{l}\text { Oxygen saturation on room air, } \\
\text { at sea level, \% }\end{array}$ & $<95$ \\
\hline Oliguria, $\mathrm{mL} / \mathrm{h}$ for $\geq 2 \mathrm{~h}^{\mathrm{a}}$ & $<35$ \\
\hline $\begin{array}{l}\text { Maternal agitation, confusion, } \\
\text { unresponsiveness or unrelenting } \\
\text { headache, or shortness of breath } \\
\text { in patient with preeclampsia }\end{array}$ & - \\
\hline
\end{tabular}

${ }^{\mathrm{a} O m i t t e d ~ i n ~ o u r ~ p a t i e n t ~ p o p u l a t i o n . ~}$

Source: Mhyre et al. ${ }^{9}$ electronic medical record. Evaluated diagnoses included pyelonephritis in pregnancy or infections of the kidney (International Classification of Diseases [ICD]-10 code 0230.00-.03 and ICD-9 code 646.6). The 6-year time frame produced a convenience sample based on the presence of an electronic medical record. Power analysis was not performed.

Members of the investigative team reviewed the medical record, ensuring that the patients were pregnant, between the ages of 18 and 50 years, and with an established clinical diagnosis of pyelonephritis. Those who did not meet these criteria were excluded. The patients were then divided into two groups: patients without a documented fever within 1 hour of initial presentation to the emergency room (ER) or labor and delivery (L\&D) triage, and patients with a documented fever within 1 hour of initial presentation. Fever was defined as a temperature greater than or equal to $38^{\circ} \mathrm{C}\left(100.4^{\circ} \mathrm{F}\right)$.

The inpatient and outpatient medical records were reviewed for basic demographic information to include age, race, parity, and body mass index (BMI) (Essentris, CliniComp, Intl., San Diego, CA). Additional data collection included the presence of comorbid medication conditions such as anemia and diabetes mellitus, and risk factors for pyelonephritis including urinary tract infection in current pregnancy, asymptomatic bacteriuria, nephrolithiasis, or sexually transmitted infection in current pregnancy. Details on vital signs from hospital admission and level of consciousness, maximum temperature during hospitalization, length of hospital stay in days, ICU admission, presence of ARDS, and preterm birth were collected. The record was additionally reviewed for results of urine culture admission. The collected data were deidentified and stored in a password protected database. STROBE guidelines were applied in preparing this article.

\section{Statistical Analysis}

Statistical analysis was performed using Stata (Version 14.2, College City, TX). Demographic data, comorbidities, clinical parameters, and outcomes were compared between the two groups using Pearson's chi-squared test or Fisher's exact to analyze categorical variables as appropriate. Student's t-test, Mann-Whitney's U-test, and Pearson's correlation coefficient were used as appropriate to analyze continuous variables. When missing data were present, an attempt was made to verify with the electronic record. Confirmed missing values were omitted from analysis. Multivariable logistic regression was used to evaluate associations between ICU admission and positive urine culture with other variables including fever and MEWC. To limit overfitting, only variables with a $p$-value $<0.25$ on bivariate analysis were included in the model. A $p$-value of $<0.05$ and a $95 \%$ confidence interval $(\mathrm{CI})$ that did not cross 1 were considered statistically significant.

\section{Results}

There were $\sim 18,800$ deliveries during the studied time period with 110 patients included in the final analysis for a rate of 5.9 cases per 1,000 deliveries. A total of 138 subjects were initially identified, with 28 cases excluded (not 


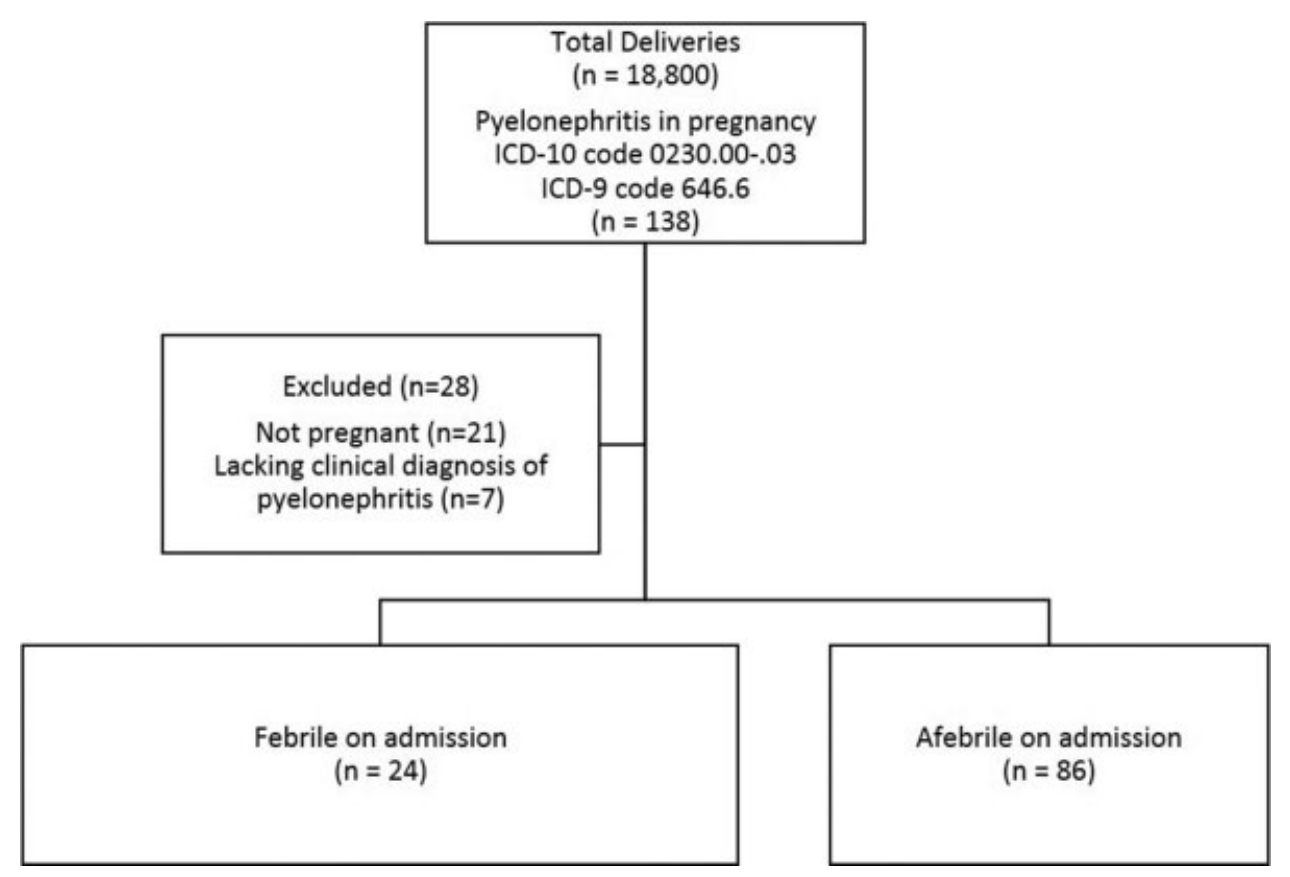

Fig. 1 Study design.

pregnant or did not have diagnosis of pyelonephritis). Of the 110 subjects meeting inclusion criteria, 24 patients were febrile on admission (21.8\%) and 86 patients were afebrile (-Fig. 1). - Table 2 demonstrates the patient demographics, and -Table 3 illustrates comorbidities and risk factors for pyelonephritis. There was no difference in age, BMI, rates of nulliparity, or racial distribution. The average gestational age on admission was 22 and 23.6 weeks for the febrile and afebrile groups, respectively ( - Table 2 ). There was no differ-

Table 2 Patient demographics

\begin{tabular}{|c|c|c|c|}
\hline & $\begin{array}{l}\text { Fever } \\
(n=24)\end{array}$ & $\begin{array}{l}\text { No fever } \\
(n=86)\end{array}$ & $p$-Value \\
\hline Age $(y)$ & $22.7 \pm 3.3$ & $24.6 \pm 4.9$ & 0.14 \\
\hline BMI & $24.8 \pm 6.9$ & $27.3 \pm 6.7$ & 0.12 \\
\hline Nulliparous & $17(70.8 \%)$ & $53(61.6 \%)$ & 0.41 \\
\hline \multicolumn{4}{|l|}{ Race } \\
\hline Caucasian & $14(60.9 \%)$ & $53(61.6 \%)$ & 0.95 \\
\hline African American & $4(17.4 \%)$ & $14(16.3 \%)$ & \\
\hline $\begin{array}{l}\text { Asian/Pacific } \\
\text { Islander }\end{array}$ & $1(4.4 \%)$ & $5(15.1 \%)$ & \\
\hline Hispanic & $1(4.4 \%)$ & $1(3.5 \%)$ & \\
\hline Other & $3(13.0 \%)$ & $13(15.1 \%)$ & \\
\hline $\begin{array}{l}\text { Estimated } \\
\text { gestational } \\
\text { age at admission } \\
\text { (wk) }\end{array}$ & $\begin{array}{l}22 \\
(17.3-28.9)\end{array}$ & $\begin{array}{l}23.6 \\
(19.1-28.3)\end{array}$ & 0.41 \\
\hline
\end{tabular}

Abbreviation: BMI, body mass index.

Note: Dichotomous variables are presented as number (\%). Continuous variables are expressed as mean \pm standard deviation or median (interquartile range). ence in the prevalence of risk factors between the two groups. The most commonly identified risk factor was prior urinary tract infection in current pregnancy with rates of 25 and $37.2 \%$ in the febrile and afebrile groups, respectively (-Table 3).

Eighteen (75\%) of the 24 febrile patients and 25 (29\%) of the 86 afebrile patients reported a fever prior to their presentation to ER or L\&D. Of the afebrile patients, 25 (29\%) developed a fever during the course of their admission. There was no difference in rates of positive urine culture between the two groups. On average, patients who were febrile on admission spent 3 days in the hospital, while afebrile patients spent only 2 days in the hospital ( $p=$ 0.004) (-Table 4)

Table 3 Comorbidities and risk factors for pyelonephritis

\begin{tabular}{|l|l|l|l|}
\hline & $\begin{array}{l}\text { Fever } \\
(\boldsymbol{n}=\mathbf{2 4 )})\end{array}$ & $\begin{array}{l}\text { No fever } \\
(\boldsymbol{n}=\mathbf{8 6})\end{array}$ & $p$-Value \\
\hline Anemia & $3(12.5)$ & $7(8.1)$ & 0.39 \\
\hline Diabetes mellitus & $0(0)$ & $6(7.0)$ & - \\
\hline Nephrolithiasis & $1(4.2)$ & $6(7.0)$ & 0.62 \\
\hline Asymptomatic bacteriuria & $1(4.2)$ & $5(5.8)$ & 0.76 \\
\hline $\begin{array}{l}\text { Urinary tract infection } \\
\text { during current pregnancy }\end{array}$ & $6(25.0)$ & $32(37.2)$ & 0.27 \\
\hline $\begin{array}{l}\text { Recurrent urinary } \\
\text { tract infections }\end{array}$ & $1(4.2)$ & $5(5.8)$ & 0.76 \\
\hline Prior pyelonephritis & $1(4.2)$ & $6(7.0)$ & 0.62 \\
\hline $\begin{array}{l}\text { Sexually transmitted } \\
\text { infection during current } \\
\text { pregnancy }\end{array}$ & $1(4.2)$ & $2(2.3)$ & 0.61 \\
\hline
\end{tabular}

Note: Values are expressed as number (\%). 
Table 4 Hospital course

\begin{tabular}{|l|l|l|l|}
\hline & Fever $(\boldsymbol{n}=\mathbf{2 4})$ & No fever $(\boldsymbol{n}=\mathbf{8 6})$ & $\boldsymbol{p}$-Value \\
\hline Admission temperature $\left({ }^{\circ} \mathrm{F}\right)$ & $101(100.6-102.1)$ & $98.5(98-99.1)$ & $<0.001$ \\
\hline Admission heart rate (beats per min) & $123(104-130)$ & $98(88-113)$ & $<0.001$ \\
\hline Maximum temperature during hospital course $\left({ }^{\circ} \mathrm{F}\right)$ & $102.4(101.4-102.9)$ & $98.9(98.7-101.5)$ & $<0.001$ \\
\hline Admission respiratory rate & $20(18-20)$ & $18(16-18)$ & 0.003 \\
\hline Admission systolic blood pressure & $114(103-121)$ & $115(108-125)$ & 0.4 \\
\hline Admission diastolic blood pressure & $67(57-70)$ & $69(63-77)$ & 0.02 \\
\hline Admission oxygen saturation $(\%)$ & $99(98.8-100)$ & $99(98-100)$ & 0.63 \\
\hline Positive maternal early warning criteria & $12(50)$ & $9(10.5)$ & $<0.001$ \\
\hline Fever during hospital course & $24(100)$ & $25(29)$ & $<0.001$ \\
\hline Reported fever at home & $18(75)$ & $25(29)$ & $<0.001$ \\
\hline Positive urine culture & $15(62.5)$ & $36(41.9)$ & 0.11 \\
\hline Days in hospital & $3(2-5)$ & $3(2-3)$ & 0.004 \\
\hline ICU admission & $2(8.3)$ & $2(2.5)$ & 0.32 \\
\hline ARDS & $2(8.3)$ & 0.19 \\
\hline
\end{tabular}

Abbreviations: ARDS, acute respiratory distress syndrome; ICU, intensive care unit.

Note: Dichotomous variables are presented as number (\%). Continuous variables are expressed as median (interquartile range).

The group of patients presenting with a fever were more likely to be tachycardic, with an average heart rate of 123 beats per minute on presentation compared with 98 beats per minute $(p<0.001)$. There were no additional clinically significant differences in admission vital signs between the two groups. However, 12 (50\%) of the febrile patients were positive for the MEWC based on their admission vital signs, while only 9 (10.5\%) of the afebrile group were positive (-Table 4 ).

A total of five patients (4.5\%) required ICU admission, three of which were afebrile on admission; four of the patients requiring ICU admission developed ARDS (3.6\%), two of which were afebrile on admission. All patients requiring ICU admission had a positive urine culture and ultimately developed a fever during their hospitalization (-Table 5). Although delivery data were available for only 89 of the 110 subjects included, the average gestational age of delivery was 39 weeks for both groups, with no difference in the rates of preterm delivery or cesarean section (-Table 6). While fever on admission was not predictive of ICU admission, positive
MEWC was with an adjusted odds ratio of 16.54 (1.29-212.5; $p=0.03$ ), when controlling for age, BMI, and concurrent diagnosis of nephrolithiasis ( - Table 7 ).

\section{Discussion}

Prior studies of pyelonephritis vary in criteria for diagnosis, specifically regarding the necessity of fever for the diagnosis. ${ }^{10}$ Fever has been shown to have a high positive predictive value in the diagnosis of pyelonephritis. ${ }^{11}$ There was no difference in the rates of ICU admission or ARDS between patients who presented with a fever compared with those who presented without a fever. Notably, three patients who were afebrile on admission ultimately required ICU admission. However, all patients who required ICU admission and developed ARDS were febrile during the course of their hospitalization. This provides evidence that while fever remains an important clinical criterion for the diagnosis of pyelonephritis, at the time of presentation, this may not be present. Thus, the

Table 5 Patients requiring ICU admission

\begin{tabular}{|c|c|c|c|c|c|c|c|c|}
\hline & $\begin{array}{l}\text { Gestational } \\
\text { age }\end{array}$ & $\begin{array}{l}\text { Fever on } \\
\text { admission } \\
+I-\end{array}$ & $\begin{array}{l}\text { Reported fever } \\
\text { prior to } \\
\text { admission }+1-\end{array}$ & $\begin{array}{l}\text { Maximum } \\
\text { temperature } \\
\text { during } \\
\text { hospitalization }\left({ }^{\circ} \mathrm{F}\right)\end{array}$ & $\begin{array}{l}\text { ARDS } \\
+1-\end{array}$ & $\begin{array}{l}\text { Positive } \\
\text { urine } \\
\text { culture } \\
+I-\end{array}$ & $\begin{array}{l}\text { Length of } \\
\text { hospitalization } \\
\text { (d) }\end{array}$ & $\begin{array}{l}\text { Gestational age } \\
\text { at the time of } \\
\text { delivery } \\
(w k+d)\end{array}$ \\
\hline 1 & $23+1$ & - & - & 102.9 & + & + & 16 & $23+3$ \\
\hline 2 & $26+2$ & - & - & 103.0 & - & + & 2 & $37+4$ \\
\hline 3 & $35+5$ & + & - & 103.0 & + & + & 8 & $35+6$ \\
\hline 4 & $21+0$ & - & - & 102.1 & - & + & 4 & $N / A^{a}$ \\
\hline 5 & $24+2$ & + & + & 102.4 & + & + & 6 & $40+3$ \\
\hline
\end{tabular}

Abbreviations: ARDS, acute respiratory distress syndrome; ICU, intensive care unit; N/A, not available.

a Patient delivered at out of network hospital, delivery data unavailable. 
Table 6 Delivery outcomes ${ }^{\mathrm{a}}$

\begin{tabular}{|l|l|l|l|}
\hline & Fever & No fever & $p$-Value \\
\hline Estimated gestational age at delivery $(w k)$ & $39.3(37.7-40.4)$ & $39(37.6-39.9)$ & 0.25 \\
\hline Cesarean delivery & 4 of $19(21.1 \%)$ & 22 of $69(31.9 \%)$ & 0.38 \\
\hline Preterm delivery & 2 of $19(10.5 \%)$ & 11 of $69(15.9 \%)$ & 0.56 \\
\hline
\end{tabular}

${ }^{\mathrm{a} D e l i v e r y}$ outcomes available 89 of 110 total patients.

Table 7 Predictors of intensive care unit admission

\begin{tabular}{|l|l|l|l|l|}
\hline & Odds ratio $(95 \% \mathrm{Cl})$ & $p$-Value & Adjusted Odds ratio $(95 \% \mathrm{Cl})^{\mathrm{a}}$ & $\boldsymbol{p}^{-V a l u e}$ \\
\hline Fever at admission & $2.48(0.39-15.8)$ & 0.32 & $1.37(0.12-15.1)$ & 0.80 \\
\hline Maternal early warning criteria & $7.68(1.19-49.47)$ & 0.03 & $16.54(1.29-212.5)$ & 0.03 \\
\hline
\end{tabular}

Abbreviations: $\mathrm{BMI}$, body mass index; $\mathrm{Cl}$, confidence interval.

${ }^{a}$ Controlled for age, BMI, and nephrolithiasis.

absence of fever does not ensure the absence of upper urinary tract infection.

There are multiple reasons why a patient may be afebrile at the time of initial evaluation. Fever may not yet be present, as is evidenced here. All of the patients who were afebrile on admission and ultimately required ICU admission did not even report a fever prior to presentation. Typically, fever is intermittent and self-administrations of analgesics or antipyretics, such as acetaminophen, may have occurred prior to presentation. Twenty-nine per cent of patients who were afebrile at presentation reported a fever prior to presentation. Given the nonnegligible risk of significant morbidity in patients who present afebrile, alternative clinical symptoms of pyelonephritis such as costovertebral angle tenderness, flank pain, nausea, or vomiting, with positive urinalysis findings warrant close observation and treatment.

Multiple other specialties have utilized early warning systems to identify those at risk of becoming critically ill with the goal of improving the opportunity for early intervention and to decrease morbidity. ${ }^{12}$ Early warning systems that have been validated in the general population, such as the systemic inflammatory response syndrome and modified early warning score, fail to effectively identify obstetric patients at risk of increased morbidity. ${ }^{13}$ It is suspected that this is due to the fact that these warning systems fail to take into consideration normal physiologic changes in pregnancy. Therefore, obstetric specific warning systems have been proposed which include the modified early obstetric warning score (MEOWS) and the MEWC to detect patients with abnormalities in clinical parameters that are associated with severe maternal morbidity and mortality, to include sepsis. The MEOWS scoring system assigns a score based on multiple parameters and the severity of the abnormality, compared with the MEWC, which utilizes single parameters to trigger further evaluation. $9,12,14$

A multicenter retrospective cohort study published by Valent et al in 2017 utilized the Acute Physiology and Chronic Health Evaluation II and MEOWS to predict prolonged hospitalization and maternal morbidity in pregnant women admitted with pyelonephritis. They found that the positive predictive value of these scores was low for adverse outcomes in patients with pyelonephritis in pregnancy. However, they still have utility in care optimization, specifically by improving communication and expediting therapeutic interventions such as antibiotic administration, thereby decreasing severe morbidity. ${ }^{15}$

The MEWC, as proposed in 2014, represents a simplified version of the MEOWs. It is important to note that fever was purposefully excluded in these criteria given that fever is unlikely to be overlooked and is often accompanied by other, more clinically relevant, vital sign abnormalities. ${ }^{9}$ Oliguria is included in MEWC; this was omitted in our study given that urine output is not available as part of an assessment at the time of a patient's initial presentation. This would, however, be useful to monitor once the patient transitions to the inpatient setting. Our study is the first to date to utilize the MEWC in the assessment of patients with pyelonephritis. Positive screening with MEWC indicated an increased risk of ICU admission. Therefore, it is prudent to consider a higher level of observation or increased frequency in monitoring these patients, as is intended by the use of an early warning system.

The overall rate of positive urine culture in our population was $46 \%$, which is lower than that which was previously reported in a similar population. ${ }^{16}$ Isolated microbial species were consistent with those previously reported; the most common was E. coli, followed by Enterococcus and Klebsiella. ${ }^{15,16}$ Clean catch specimen collection is the standard practice at our facility, which likely contributed to a high rate of mixed flora results with contaminated specimens. We did not take into account timing of antibiotic administration or prior antibiotic use in the collection of these urine cultures, both of which may lead to false-negative urine cultures. This relatively low rate of positive urine cultures leads to the concern for overdiagnosis of pyelonephritis in our patient population; however, given that the rate of pyelonephritis in our population was similar to previously reported rates, this is unlikely. It is important to consider pyelonephritis in the initial differential diagnosis of patients with appropriate symptoms, and empiric treatment 
must begin in advance of urine culture results. Culture results are most useful to guide antibiotic therapy for the completion of treatment and prophylaxis. However, due to the delay in results, urine cultures have previously been shown to be of limited clinical utility in guiding the clinical management of pregnant women with pyelonephritis. ${ }^{16}$

Our study was conducted at a single institution, potentially limiting its generalizability. Additional limitations include the reliance on the electronic medical record for the identification of patients and collection of data. With use of ICD coding, there is concern that some charts may have been improperly coded. We accounted for this with detailed review of the medical record to ensure veracity of the diagnosis of pyelonephritis, with exclusion of patients without a clinical diagnosis of pyelonephritis despite ICD coding. However, the possibility remains that some cases may not have been captured. Our rate of pyelonephritis, ARDS, and ICU admission are all similar to that which has previously been reported. With rare adverse events, a larger sample size would afford improved ability to assess the predictive nature of the MEWC score in predicting maternal morbidity in the setting of pyelonephritis.

\section{Conclusion}

Pyelonephritis is associated with significant maternal and neonatal morbidities and early identification of those patients at risk may lead to improved outcomes. Patients who are afebrile at the time of admission are still at risk for significant morbidity with similar rates of ICU admission, pulmonary edema, ARDS, and preterm delivery as febrile patients. Therefore, in patients with clinical symptoms and urinalysis findings suggestive of pyelonephritis, even in the absence of fever, we recommend continuing the practice of hospital admission for intravenous antibiotics and close observation. Patients with the presence of MEWC are at increased risk of maternal morbidity in the setting of pyelonephritis in pregnancy; therefore, consideration should be given for increased frequency or level of monitoring for early identification of a deterioration in their clinical status.

Note

The views expressed in this article reflect the results of research conducted by the authors and do not necessarily reflect the official policy or position of the Department of the Navy, Department of Defense, or the United States government.

I am a military service member. This work was prepared as part of my official duties. Title 17 U.S.C. 105 provides that "Copyright protection under this title is not available for any work of the United States government." Title 17 U.S.C. 101 defines a United States government work as a work prepared by a military service member or employee of the United States government as part of that person's official duties.
The study protocol was approved by the Naval Medical Center Portsmouth Institutional Review Board in compliance with all applicable Federal regulations governing the protection of human subjects.

Research data derived from an approved Naval Medical Center, Portsmouth, Virginia IRB, protocol; number NMCP.2017.0061.

\section{Conflict of Interest}

None.

\section{Acknowledgment}

We would like to thank Ms. Donna Eastham, BA, for her assistance with article editing and preparation.

\section{References}

1 Wing DA, Fassett MJ, Getahun D. Acute pyelonephritis in pregnancy: an 18-year retrospective analysis. Am J Obstet Gynecol 2014;210(03):219.e1-219.e6

2 Say L, Chou D, Gemmill A, et al. Global causes of maternal death: a WHO systematic analysis. Lancet Glob Health 2014;2(06): e323-e333

3 Hill JB, Sheffield JS, McIntire DD, Wendel GD Jr. Acute pyelonephritis in pregnancy. Obstet Gynecol 2005;105(01):18-23

4 Foxman B. Epidemiology of urinary tract infections: incidence, morbidity, and economic costs. Am J Med 2002;113(Suppl 1A):5S-13S

5 Glaser AP, Schaeffer AJ. Urinary tract infection and bacteriuria in pregnancy. Urol Clin North Am 2015;42(04):547-560

6 Gilstrap LC III, Ramin SM. Urinary tract infections during pregnancy. Obstet Gynecol Clin North Am 2001;28(03):581-591

7 Le Gouez A, Benachi A, Mercier FJ. Fever and pregnancy. Anaesth Crit Care Pain Med 2016;35(Suppl 1):S5-S12

8 Barton JR, Sibai BM. Severe sepsis and septic shock in pregnancy. Obstet Gynecol 2012;120(03):689-706

9 Mhyre JM, D’Oria R, Hameed AB, et al. The maternal early warning criteria: a proposal from the national partnership for maternal safety. Obstet Gynecol 2014;124(04):782-786

10 Gilstrap LC III, Cunningham FG, Whalley PJ. Acute pyelonephritis in pregnancy: an anterospective study. Obstet Gynecol 1981;57 (04):409-413

11 Pinson AG, Philbrick JT, Lindbeck GH, Schorling JB. Fever in the clinical diagnosis of acute pyelonephritis. Am J Emerg Med 1997; 15(02):148-151

12 Friedman AM. Maternal early warning systems. Obstet Gynecol Clin North Am 2015;42(02):289-298

13 Lappen JR, Keene M, Lore M, Grobman WA, Gossett DR. Existing models fail to predict sepsis in an obstetric population with intrauterine infection. Am J Obstet Gynecol 2010;203(06):573. e1-573.e5

14 Chebbo A, Tan S, Kassis C, Tamura L, Carlson RW. Maternal sepsis and septic shock. Crit Care Clin 2016;32(01):119-135

15 Valent AM, Peticca K, DiMatteo A, et al. Pyelonephritis in pregnancy: prediction of prolonged hospitalization and maternal morbidity using prognostic scoring systems. Am J Perinatol 2017;34(12):1212-1218

16 Wing DA, Park AS, Debuque L, Millar LK. Limited clinical utility of blood and urine cultures in the treatment of acute pyelonephritis during pregnancy. Am J Obstet Gynecol 2000;182(06): $1437-1440$ 